\title{
Breakdown of the Peierls substitution for the Haldane model with ultracold atoms
}

\author{
Julen Ibañez-Azpiroz, ${ }^{1}$ Asier Eiguren, ${ }^{2,3}$ Aitor Bergara, ${ }^{2,3,4}$ Giulio Pettini, ${ }^{5}$ and Michele Modugno ${ }^{6,7}$ \\ ${ }^{1}$ Peter Grünberg Institute and Institute for Advanced Simulation, Forschungszentrum Jülich and JARA, D-52425 Jülich, Germany \\ ${ }^{2}$ Departamento de Física de la Materia Condensada, Universidad del Pais Vasco, UPV/EHU, 48080 Bilbao, Spain \\ ${ }^{3}$ Donostia International Physics Center, 20018 Donostia, Spain \\ ${ }^{4}$ Centro de Física de Materiales, Centro Mixto CSIC-UPV/EHU, 20018 Donostia, Spain \\ ${ }^{5}$ Dipartimento di Fisica e Astronomia, Università di Firenze and INFN, 50019 Sesto Fiorentino, Italy \\ ${ }^{6}$ Departamento de Física Teórica e Histórico de la Ciencia, Universidad del Pais Vasco, UPV/EHU, 48080 Bilbao, Spain \\ ${ }^{7}$ Ikerbasque, Basque Foundation for Science, 48011 Bilbao, Spain \\ (Received 15 May 2014; published 8 September 2014)
}

\begin{abstract}
We present two independent approaches for calculating the tight-binding parameters of the Haldane model with ultracold atoms. The tunneling coefficients up to next-nearest neighbors are computed ab initio by using the maximally localized Wannier functions and compared to analytical expressions written in terms of gauge-invariant measurable properties of the spectrum. The two approaches present a remarkable agreement and evidence the breakdown of the Peierls substitution: (i) the phase acquired by the next-nearest tunneling amplitude $t_{1}$ presents quantitative and qualitative differences with respect to that obtained by the integral of the vector field $\boldsymbol{A}$, as considered in the Peierls substitution, even in the regime of low amplitudes of $\boldsymbol{A}$; and (ii) for larger values, also $\left|t_{1}\right|$ and the nearest-neighbor tunneling $t_{0}$ have a marked dependence on $\boldsymbol{A}$. The origin of this behavior and its implications are discussed.
\end{abstract}

DOI: 10.1103/PhysRevA.90.033609

PACS number(s): 67.85.- d, 73.43.-f

\section{INTRODUCTION}

The Haldane model is a celebrated two-dimensional periodic tight-binding model, characterized by a quantum Hall effect caused by the breaking of time-reversal symmetry with zero magnetic flux through the unit cell [1]. Recently, there have been proposals for engineering the Haldane model with ultracold atoms in optical lattices by means of artificial gauge fields $[2,3]$ and to study the associated topological quantum states in the presence of sharp boundaries [4]. In fact, these systems represent a very interesting platform for simulating solid-state physics [5].

The Haldane model is characterized by exotic quantum phases, with different Chern numbers, depending on the value of the phase $\varphi$ of the next-nearest tunneling amplitude $t_{1}$. In the contemporary literature, a very popular approximation for estimating the effect of the vector potential $\boldsymbol{A}$ on the tunneling coefficients is the so-called Peierls substitution, in which the tight-binding coefficients $t_{i j}$ are simply replaced by $t_{i j} \exp \left\{i e \int_{i}^{j} \boldsymbol{A} \cdot d \boldsymbol{r}\right\}[6-21]$. Other approaches make use of a simplified basis of localized functions, constructed by means of approximate atomic orbitals [4].

In this paper we go beyond these approximate methods and present two independent calculations of the tight-binding parameters for the Haldane model discussed in Refs. [2,4]. In particular, we show that, within the next-nearest neighbors approximation, the tunneling coefficients can be directly written in terms of gauge-invariant measurable properties of the spectrum (namely, the gap at the Dirac point and the bandwidths) or computed ab initio by using the maximally localized Wannier functions (MLWFs) [22,23]. The real character of the nearest-neighbor tunneling $t_{0}$ amplitude and the phase structure of the next-nearest-neighbor tunneling $t_{1}$ are obtained as a direct consequence of the symmetries of the lattice. Notably, the two approaches present a remarkable agreement and evidence the breakdown of the Peierls substitution. As a matter of fact, we advance that the phase acquired by the next-nearest tunneling amplitude $t_{1}$ is quantitatively different from that predicted by the Peierls substitution and presents a pronounced dependence on the intensity of the underlying scalar potential. Moreover, both the amplitudes of $t_{0}$ and $t_{1}$ turn out to be dependent on the intensity of $\boldsymbol{A}$, which is contrary to the assumptions made behind the Peierls substitution.

\section{MODEL}

Let us start from the following single-particle, minimalcoupling Hamiltonian in two dimensions:

$$
\hat{H}_{0}=\frac{1}{2 m}[\hat{\boldsymbol{p}}-\boldsymbol{A}(\boldsymbol{r})]^{2}+V_{L}(\boldsymbol{r}),
$$

with $\boldsymbol{r}=(x, y), \boldsymbol{p}=-i \hbar \nabla$, and $V_{L}(\boldsymbol{r})$ being the following honeycomb potential $[4,23,24]$ :

$V_{L}(\boldsymbol{r})=s E_{R}\left\{2 \cos \left[\left(\boldsymbol{b}_{1}-\boldsymbol{b}_{2}\right) \cdot \boldsymbol{r}\right]+2 \sum_{i=1}^{2} \cos \left(\boldsymbol{b}_{i} \cdot \boldsymbol{r}\right)\right\}$,

where the vectors $\boldsymbol{b}_{1 / 2}=\left(\sqrt{3} / 2 k_{L}\right)\left(\boldsymbol{e}_{x} \mp \sqrt{3} \boldsymbol{e}_{y}\right)$ generate the reciprocal lattice, $k_{L}$ is the laser wavelength, and $s$ is the amplitude of the potential in units of the recoil energy $E_{R}=\hbar^{2} k_{L}^{2} / 2 m$ [25]. Notice that, though this specific realization is characterized by degenerate potential wells, an imbalance can be easily produced by introducing a suitable phase [2,24]. The corresponding Bravais lattice, $\mathcal{B}=\left\{j_{1} \mathbf{a}_{1}+\right.$ $\left.j_{2} \mathbf{a}_{2} \mid j_{1}, j_{2}=0, \pm 1, \pm 2 \ldots\right\}$, with lattice constant $a$ [such that $k_{L}=4 \pi /(3 \sqrt{3} a)$ [24]], is generated by the two basis vectors $\boldsymbol{a}_{1 / 2}=\left(2 \pi / 3 k_{L}\right)\left(\boldsymbol{e}_{x}, \mp \sqrt{3} \boldsymbol{e}_{y}\right)$, obeying $\boldsymbol{a}_{i} \cdot \boldsymbol{b}_{j}=2 \pi \delta_{i j}$. As for the vector potential, we consider the same expression discussed in Refs. [2,4] [corresponding to the Coulomb gauge, 

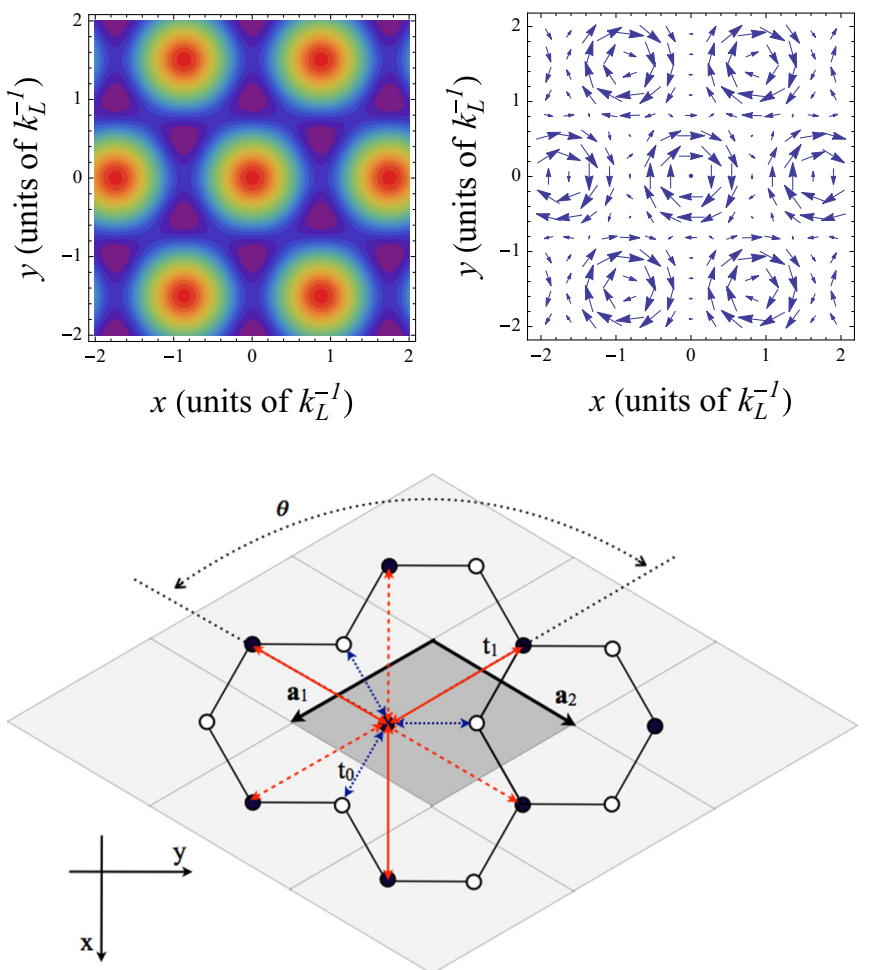

FIG. 1. (Color online) Top: Structure of the scalar (left) and vector (right) potentials. In the left panel, hot and cold colors correspond to maxima and minima of the potential, respectively. Bottom: Bravais lattice associated to the honeycomb potential in Eq. (2). Black and white circles refers to minima of type $A$ and $B$, respectively. The elementary cell is highlighted in gray. The various tunneling coefficients are indicated for the site of type $A$ in the central cell. The system is invariant under discrete translation generated by the Bravais vectors $\boldsymbol{a}_{1 / 2}$ and under rotations by $\theta=2 \pi / 3$ radians around any vertex of the lattice. The former implies that next-nearest tunneling amplitudes $t_{1}$ along the same direction are conjugate pairs (solid and dashed lines); from the latter follows the equivalence of the hopping amplitudes separated by $2 \pi / 3$ radians. When sites $A$ and $B$ are degenerate, the system is also invariant under rotations by $\pi$ radians around the center of any elementary cell; this implies that $t_{0}$ is real.

$$
\begin{aligned}
\nabla \cdot \boldsymbol{A}(\boldsymbol{r})=0]: \\
\begin{aligned}
\boldsymbol{A}(\boldsymbol{r})= & \alpha \hbar k_{L}\left(\left\{\sin \left[\left(\boldsymbol{b}_{2}-\boldsymbol{b}_{1}\right) \cdot \boldsymbol{r}\right]+\frac{1}{2} \sin \left(\boldsymbol{b}_{2} \cdot \boldsymbol{r}\right)\right.\right. \\
& \left.\left.-\frac{1}{2} \sin \left(\boldsymbol{b}_{1} \cdot \boldsymbol{r}\right)\right\} \boldsymbol{e}_{x}-\frac{\sqrt{3}}{2}\left[\sin \left(\boldsymbol{b}_{1} \cdot \boldsymbol{r}\right)+\sin \left(\boldsymbol{b}_{2} \cdot \boldsymbol{r}\right)\right] \boldsymbol{e}_{y}\right),
\end{aligned}
\end{aligned}
$$

that has the same symmetry as the underlying honeycomb potential (see Fig. 1). The parameter $\alpha$ represents the amplitude of the vector potential in units of $\hbar k_{L}$.

\section{TIGHT-BINDING EXPANSION}

The tight-binding model is constructed from the manybody Hamiltonian $\hat{\mathcal{H}}_{0}=\int d \boldsymbol{r} \hat{\psi}^{\dagger}(\boldsymbol{r}) \hat{H}_{0} \hat{\psi}(\boldsymbol{r})$, by expanding the field operator on a basis of localized functions, $\hat{\psi}(\boldsymbol{r}) \equiv \sum_{j v} \hat{a}_{\boldsymbol{j} v} w_{\boldsymbol{j} v}(\boldsymbol{r})$, with the usual commutation rules $\left[\hat{a}_{j v}, \hat{a}_{j^{\prime} v^{\prime}}^{\dagger}\right]=\delta_{j j^{\prime}} \delta_{v v^{\prime}}$. Then, by restricting the analysis to the two lowest bands, $\hat{\mathcal{H}}_{0}$ can be written as [23]

$$
\hat{\mathcal{H}}_{0}=\sum_{\nu \nu^{\prime}=A, B} \sum_{\boldsymbol{j}, \boldsymbol{j}^{\prime}} \hat{a}_{\boldsymbol{j} v}^{\dagger} \hat{a}_{\boldsymbol{j}^{\prime} v^{\prime}}\left\langle w_{\boldsymbol{j} v}\left|\hat{H}_{0}\right| w_{\boldsymbol{j}^{\prime} v^{\prime}}\right\rangle,
$$

where the matrix elements $\left\langle w_{j v}\left|\hat{H}_{0}\right| w_{j^{\prime} v^{\prime}}\right\rangle$ correspond to tunneling amplitudes between different lattice sites (except for the special case $\boldsymbol{j}^{\prime}=\boldsymbol{j}, v^{\prime}=v$, representing the onsite energies). These matrix elements depend only on $\boldsymbol{j}^{\prime}-\boldsymbol{j}$ due to the translational invariance of the lattice. The spectrum of $\hat{\mathcal{H}}_{0}$ can be obtained by considering the following transformation from coordinate to momentum space, $\hat{b}_{v \boldsymbol{k}}=$ $\left(1 / \sqrt{S_{\mathcal{B}}}\right) \sum_{j} e^{-i \boldsymbol{k} \cdot \boldsymbol{R}_{j}} \hat{a}_{\boldsymbol{j} v}$, yielding

$$
\hat{\mathcal{H}}_{0}^{t b}=\sum_{\nu \nu^{\prime}=A, B} \int_{S_{\mathcal{B}}} d \boldsymbol{k} h_{\nu \nu^{\prime}}(\boldsymbol{k}) \hat{b}_{\nu \boldsymbol{k}}^{\dagger} \hat{b}_{\nu^{\prime} \boldsymbol{k}},
$$

with $h_{v v^{\prime}}(\boldsymbol{k})=\sum_{j} e^{i \boldsymbol{k} \cdot \boldsymbol{R}_{\boldsymbol{j}}}\left\langle w_{\mathbf{0} v}\left|\hat{H}_{0}\right| w_{\boldsymbol{j} v^{\prime}}\right\rangle$ and $S_{\mathcal{B}}$ indicating the first Brillouin zone [23]. By truncating the above expression to next-nearest neighbors as usual [1,2], we define

$$
h_{v v^{\prime}}(\boldsymbol{k}) \equiv\left[h_{v v}^{(0)}(\boldsymbol{k})+h_{v v}^{(2)}(\boldsymbol{k})\right] \delta_{\nu v^{\prime}}+h_{v \nu^{\prime}}^{(1)}(\boldsymbol{k}) .
$$

The first term corresponds to the onsite energies:

$$
h_{\nu v}^{(0)}(\boldsymbol{k})=\left\langle w_{\mathbf{0} v}\left|\hat{H}_{0}\right| w_{\mathbf{0} v}\right\rangle \equiv E_{\nu} .
$$

The last term has only off-diagonal elements, corresponding to the hopping toward the three nearest-neighbor sites (see Fig. 1). Thanks to the symmetries of the Hamiltonian (1), the three tunneling amplitudes are equal. By defining $t_{0} \equiv$ $\left\langle w_{\mathbf{0} A}\left|\hat{H}_{0}\right| w_{\mathbf{0}}\right\rangle$, we can write

$$
h_{12}^{(1)}(\boldsymbol{k})=t_{0}\left(1+e^{i \boldsymbol{k} \cdot \boldsymbol{a}_{1}}+e^{-i \boldsymbol{k} \cdot \boldsymbol{a}_{2}}\right) \equiv t_{0} Z_{0}(\boldsymbol{k}) \equiv z(\boldsymbol{k})
$$

and $h_{21}^{(1)}(\boldsymbol{k})=z^{*}(\boldsymbol{k})$. Finally, by defining

$$
t_{1 v}=\left\langle w_{\mathbf{0} v}\left|\hat{H}_{0}\right| w_{\left(\boldsymbol{a}_{1}+\boldsymbol{a}_{2}\right) v}\right\rangle \equiv\left|t_{1 v}\right| e^{i \varphi_{v}},
$$

and taking again into account the symmetries of the system (see Fig. 1), the second term-corresponding to next-nearest tunneling between homologous sites-can be cast in the following form:

$$
\begin{aligned}
h_{v \nu}^{(2)}(\boldsymbol{k})= & \left|t_{1 v}\right|\left\{2 \cos \left[\boldsymbol{k} \cdot\left(\boldsymbol{a}_{1}+\boldsymbol{a}_{2}\right)+\varphi_{\nu}\right]\right. \\
& \left.+2 \sum_{i=1,2} \cos \left(\boldsymbol{k} \cdot \boldsymbol{a}_{i}-\varphi_{\nu}\right)\right\} \equiv\left|t_{1 v}\right| F_{v}(\boldsymbol{k}) .
\end{aligned}
$$

Notice that in general the onsite energies and the tunneling coefficients depend on the amplitudes of both the scalar and vector potentials: $E_{v}=E_{v}(s, \alpha), t_{0}=t_{0}(s, \alpha),\left|t_{1}\right|=\left|t_{1}\right|(s, \alpha)$, $\varphi_{\nu}=\varphi_{\nu}(s, \alpha)$. This is a direct consequence of the fact that the optimal choice for the basis of localized functions $w_{j v}(\boldsymbol{r})$ depends on the properties of the overall structure of the Hamiltonian (1). By defining

$$
\epsilon_{v}(\boldsymbol{k})=E_{v}+\left|t_{1 v}\right| F_{v}(\boldsymbol{k}),
$$

we can write

$$
h_{v \nu^{\prime}}(\boldsymbol{k})=\left(\begin{array}{cc}
\epsilon_{A}(\boldsymbol{k}) & z(\boldsymbol{k}) \\
z^{*}(\boldsymbol{k}) & \epsilon_{B}(\boldsymbol{k})
\end{array}\right),
$$


that is equivalent to the expression discussed in Ref. [2]. However, we remark that here we have not made explicit use of the Peierls substitution and that the dependence of Eq. (10) on the phase $\varphi_{\nu}$ is a consequence of the symmetries of the full potential.

Finally, by diagonalizing the matrix $h_{v v^{\prime}}(\boldsymbol{k})$ and defining $f_{ \pm}(\boldsymbol{k}) \equiv\left(\left|t_{1 A}\right| F_{A}(\boldsymbol{k}) \pm\left|t_{1 B}\right| F_{B}(\boldsymbol{k})\right) / 2$, we get the following expression for the spectrum of the lowest two bands:

$$
\epsilon_{ \pm}(\boldsymbol{k})=f_{+}(\boldsymbol{k}) \pm \sqrt{\left|\epsilon+f_{-}(\boldsymbol{k})\right|^{2}+|z(\boldsymbol{k})|^{2}},
$$

that is a function of $\left|t_{0}\right|,\left|t_{1 v}\right|$, and $\varphi_{\nu}$.

\section{RESULTS}

In the following we will consider for simplicity the degenerate case $\epsilon=0 \quad\left(E_{A}=E_{B}\right)$, corresponding to the potential in Eq. (2). In this case, thanks to the symmetries of the system, we have $\left|t_{1 A}\right|=\left|t_{1 B}\right| \equiv\left|t_{1}\right|, \varphi_{A}=-\varphi_{B} \equiv \varphi$, and $\left\langle w_{\mathbf{0} A}\left|\hat{H}_{0}\right| w_{\mathbf{0} B}\right\rangle=\left\langle w_{\mathbf{0} B}\left|\hat{H}_{0}\right| w_{\mathbf{0} A}\right\rangle$ (when $A$ and $B$ are equivalent the system is invariant under rotation by $\pi$ radians around the center of any cell; see Fig. 1). The latter implies that $t_{0}$ is real. Remarkably, in this case the two tunneling amplitudes $t_{0}$ and $\left|t_{1}\right|$ and the phase $\varphi$ can be expressed in terms of specific properties of the spectrum. Let us start by noticing that $f_{+}(\mathbf{0})=6\left|t_{1}\right| \cos \varphi, f_{-}(\mathbf{0})=0,|z(\mathbf{0})|=3 t_{0}$. In addition, we indicate with $\boldsymbol{k}_{D}$ the position of the Dirac points [23] and define $\Delta_{ \pm} \equiv \pm\left[\epsilon_{ \pm}(\mathbf{0})-\epsilon_{ \pm}\left(\boldsymbol{k}_{D}\right)\right]$, that correspond to the two bandwidths when the tunneling coefficients satisfy the hierarchy $\left|t_{1}\right| \ll t_{0}$. Then, we have

$$
t_{0}=\left(\Delta_{+}+\Delta_{-}+\delta_{D}\right) / 6,
$$

with $\delta_{D} \equiv \epsilon_{+}\left(\boldsymbol{k}_{D}\right)-\epsilon_{-}\left(\boldsymbol{k}_{D}\right)$ being the gap at the Dirac points, due to the presence of the vector potential. Also, at, e.g., $\boldsymbol{k}_{D}=(1,0) k_{L}$, we have $f_{+}\left(\boldsymbol{k}_{D}\right)=-3\left|t_{1}\right| \cos \varphi, f_{-}\left(\boldsymbol{k}_{D}\right)=$ $3 \sqrt{3}\left|t_{1}\right| \sin \varphi,\left|z\left(\boldsymbol{k}_{D}\right)\right|=0$, yielding [2]

$$
\delta_{D}=6 \sqrt{3}\left|t_{1}\right| \sin \varphi .
$$

Another relation containing $\left|t_{1}\right|$ and $\varphi$ is

$$
\Delta_{+}-\Delta_{-}=18\left|t_{1}\right| \cos \varphi \text {. }
$$

Then, by combining Eqs. (15) and (16), we get

$$
\begin{aligned}
\left|t_{1}\right| & =\frac{1}{18} \sqrt{\left(\Delta_{+}-\Delta_{-}\right)^{2}+3 \delta_{D}^{2}}, \\
\varphi & =\tan ^{-1}\left[\sqrt{3} \frac{\delta_{D}}{\Delta_{+}-\Delta_{-}}\right] .
\end{aligned}
$$

Eqs. (14), (17), and (18) represent an important contribution of this work: they provide a way to connect the value of the tunneling amplitudes to gauge-invariant measurable properties of the spectrum. Moreover, they also provide a straightforward method for computing the tunneling amplitudes, as the exact Bloch spectrum can be readily computed by means of a standard Fourier decomposition [23,24], even in the presence of a vector potential [26]. We remark that, in principle, the same approach can be generalized to the nondegenerate case or to other tight-binding models.

In addition, we compare these values with those computed $a b$ initio from their definition in terms of the matrix elements $\left\langle w_{j v}\left|\hat{H}_{0}\right| w_{j^{\prime} v^{\prime}}\right\rangle$. To this end, we make use of the MLWFs for

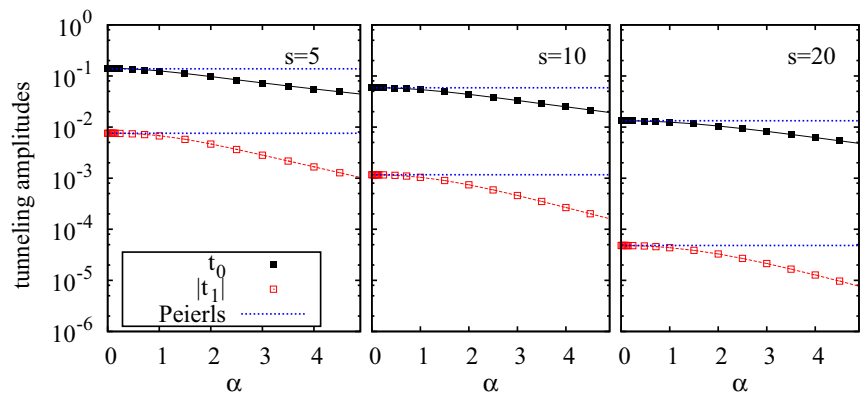

FIG. 2. (Color online) Tunneling amplitudes $t_{0}$ (black full squares) and $\left|t_{1}\right|$ (red empty squares) for $s=5,10,20$ (from left to right), as calculated from the MLWFs (points) and from the exact spectrum (lines). The agreement is remarkable. The horizontal blue (dotted) lines represent the values corresponding to the Peierls substitution. The values of the tunnelings are given in units of $E_{R}$.

composite bands [22], which are defined through the following unitary mixing of the two lowest Bloch bands:

$$
w_{\boldsymbol{j} \nu}(\boldsymbol{r})=\frac{1}{\sqrt{S_{\mathcal{B}}}} \int_{S_{\mathcal{B}}} d \boldsymbol{k} e^{-i \boldsymbol{k} \boldsymbol{R}_{j}} \sum_{m=1}^{2} U_{v m}(\boldsymbol{k}) \psi_{m \boldsymbol{k}}(\boldsymbol{r}),
$$

with $\boldsymbol{R}_{\boldsymbol{j}} \in \mathcal{B}, \psi_{m \boldsymbol{k}}$ being the eigenfunctions of the Hamiltonian (1) [8] and $U_{v m}(\boldsymbol{k})$ being $2 \times 2$ unitary matrices, which are periodic in $\boldsymbol{k}$ space and minimizing the spread of $w_{j v}(\boldsymbol{r})$ [22]. In the present case, the MLWFs are obtained by modifying the code discussed in Ref. [23] in order to include a vector potential. The MLWFs turn out to be complex due to the breaking of the time reversal symmetry, and this explains the emergence of a phase factor in the tunneling coefficients [26].

In Figs. 2 and 3 we show the behavior of $t_{0},\left|t_{1}\right|$ and $\varphi$ as a function of the amplitude $\alpha$ of the vector potential, for different values of the intensity $s$ of the honeycomb lattice. The agreement between the values obtained via the MLWFs and those extracted from the spectrum is remarkable [27]. From these figures we can identify two regimes as a function of $\alpha$ : (i) for small enough values, $\alpha \lesssim 1$, where $t_{0}$ and $\left|t_{1}\right|$ are almost constant and the phase $\varphi$ is linear in $\alpha$; and (ii) for $\alpha \gtrsim 1$ where the dependence on $\alpha$ is less trivial. In the latter

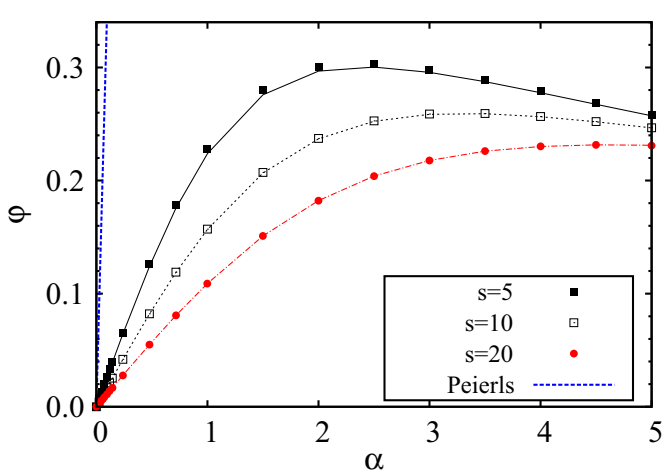

FIG. 3. (Color online) Plot of the phase $\varphi$ as a function of the amplitude $\alpha$ of the vector potential, for $s=5,10,20$, as calculated from the MLWFs (points) and from the exact spectrum (lines). The prediction of the Peierls substitution is represented by the blue (dotted) line, that is almost indistinguishable from the vertical axis. 


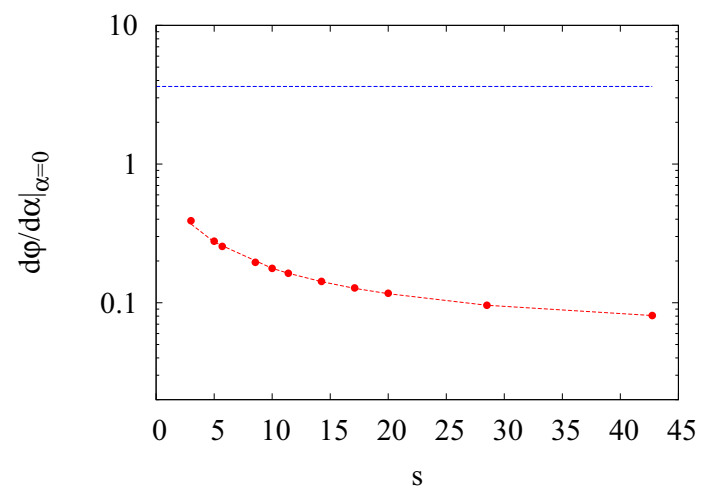

FIG. 4. (Color online) Plot of $d \varphi /\left.d \alpha\right|_{\alpha=0}$ calculated from the MLWFs (points) and from the exact spectrum (lines), as a function of the amplitude $s$ of the honeycomb potential. The horizontal dashed line represents the value corresponding to the Peierls phase $\varphi=(2 \pi / \sqrt{3}) \alpha$. Note the logarithmic scale on the vertical axis. We remark that the present tight-binding model is accurate only for $s \gtrsim 3$; for lower values it may be necessary to consider also other next-to-leading tunneling coefficients [23].

regime, $t_{0}$ and $\left|t_{1}\right|$ present a pronounced dependence on $\alpha$, in clear contrast with the Peierls substitution (see horizontal lines in Fig. 2) which assumes the phase $\varphi$ to be the only $\alpha$-dependent quantity. In addition, the phase strongly deviates from the linear behavior expected from the Peierls substitution, namely, $\varphi=\int_{\boldsymbol{r}_{A}}^{\boldsymbol{r}_{A}-\boldsymbol{a}_{1}} \boldsymbol{A} \cdot d \boldsymbol{r}=(2 \pi / \sqrt{3}) \alpha$ [2] (see Fig. 3). This figure reveals that the Peierls substitution dramatically fails even in the "linear" regime, as it predicts a slope for the phase far larger than the actual one. Moreover, it completely neglects its dependence on the amplitude $s$ of the scalar potential (that is appreciable even in the full tight-binding regime, $s>10$ ). This is particularly evident from Fig. 4, where we plot the behavior of the angular coefficient in the linear regime, $d \varphi /\left.d \alpha\right|_{\alpha=0}$, as a function of $s$. This figure provides further evidence that the Peierls substitution does not even provide a reasonable estimate for the order of magnitude of $\varphi$ in the linear regime.

\section{DISCUSSION}

The results just discussed are surprising, as the use of the Peierls substitution is well established in the literature and it has always been considered as a solid rule for constructing the Haldane model $[1,2]$. However, a more careful analysis reveals that the breakdown of the Peierls substitution in the Haldane model could have been foreseen. In fact, we recall that the Peierls substitution is valid only for slowly varying potentials $[7,10,11]$, a condition that is explicitly violated in the Haldane model, as this model requires a vector potential with the same periodicity of the underlying lattice. In particular, the rigorous demonstration of the Peierls substitution necessitates the hypothesis of a same-site, same-orbital interaction $\left\langle w_{j v}|\boldsymbol{A}(\boldsymbol{r})| w_{j^{\prime} v^{\prime}}\right\rangle=\boldsymbol{A}\left(\boldsymbol{R}_{j v}\right)\left\langle w_{j v} \mid w_{j^{\prime} v^{\prime}}\right\rangle$ [11], that cannot be satisfied here. This is related to the fact that the usual implicit assumption that the basis of localized orbitals is not affected by the vector potential (see, e.g., [11]) is not valid in the present situation. Indeed, the presence of a generic vector potential may significantly affect both the Bloch eigenfunctions $\psi_{m k}$ and the gauge transformation $U_{v m}$ entering Eq. (19). A clear consequence of this can be found in the change of magnitude of the tunneling coefficients $t_{0}$ and $t_{1}$ with respect to the intensity of the vector potential, as depicted in Fig. 2. Therefore, we conclude that in general both the scalar and vector potentials must be treated on equal footing, and all the parameters $\left(t_{0}\right.$, $\left|t_{1}\right|$, and $\varphi$ ) must be considered as dependent on both $s$ and $\alpha$. We note that similar warnings about the uncontrolled use of the Peierls substitution were already pointed out in Refs. [8,9], though in different contexts.

\section{CONCLUSIONS}

We have presented two independent approaches for calculating the tight-binding parameters for the Haldane model with ultracold atoms [2]. The first is based on ab initio MLWFs and the second in terms of gauge-invariant properties of the spectrum, summarized in Eqs. (14), (17), and (18). The latter provides a straightforward approach whenever the spectrum can be measured or computed with sufficient accuracy. The results obtained with both methods present a remarkable agreement and demonstrate the failure of the Peierls substitution in predicting quantitative and even qualitative properties of the tunneling coefficients of the Haldane model. A similar breakdown should be expected in any physical system where the vector gauge field varies on the same scale as the lattice, regardless of the system being cold atoms in optical lattices or electrons in a solid. We believe that, besides their conceptual significance, the results presented in this work are of practical relevance, as they allow for quantitative descriptions guiding the current research activity in this field.

Note added in proof. Recently, an experimental realization of the Haldane model with ultracold atoms has been reported in [28]. This experiment exploits a method for creating the artificial gauge field that is different from the one discussed in the present work.

\section{ACKNOWLEDGMENTS}

This work has been supported by the Universidad del Pais Vasco/Euskal Herriko Unibertsitatea under Programs No. UFI 11/55 and No. UFI IT-366-07, the Ministerio de Economía y Competitividad through Grants No. FIS2010-19609-C0200 and No. FIS2012-36673-C03-03, and the Basque Government through Grant No. IT-472-10. J.I.A. would like to acknowledge support from the Helmholtz Gemeinschaft Deutscher-Young Investigators Group Program No. VH-NG717 (Functional Nanoscale Structure and Probe Simulation Laboratory).
[1] F. D. M. Haldane, Phys. Rev. Lett. 61, 2015 (1988).

[2] L. B. Shao, S.-L. Zhu, L. Sheng, D. Y. Xing, and Z. D. Wang, Phys. Rev. Lett. 101, 246810 (2008).
[3] E. Anisimovas, F. Gerbier, T. Andrijauskas, and N. Goldman, Phys. Rev. A 89, 013632 (2014). 
[4] T. D. Stanescu, V. Galitski, J. Y. Vaishnav, C. W. Clark, and S. Das Sarma, Phys. Rev. A 79, 053639 (2009); T. D. Stanescu, V. Galitski, and S. Das Sarma, ibid. 82, 013608 (2010).

[5] M. Lewenstein, A. Sanpera, and V. Ahufinger, Ultracold Atoms in Optical Lattices: Simulating Quantum Many-Body Systems (Oxford University, Oxford, 2012).

[6] R. Peierls, Z. Phys. 80, 763 (1933).

[7] J. Luttinger, Phys. Rev. 84, 814 (1951).

[8] W. Kohn, Phys. Rev. 115, 1460 (1959).

[9] A. S. Alexandrov and H. Capellmann, Phys. Rev. Lett. 66, 365 (1991); Z. Phys. B 83, 237 (1991).

[10] M. Graf and P. Vogl, Phys. Rev. B 51, 4940 (1995).

[11] T. B. Boykin, R. C. Bowen, and G. Klimeck, Phys. Rev. B 63, 245314 (2001); T. B. Boykin, Am. J. Phys. 69, 793 (2001).

[12] B. A. Bernevig, Topological Insulators and Topological Superconductors (Princeton University, Princeton, NJ, 2013).

[13] N. Goldman, A. Kubasiak, A. Bermudez, P. Gaspard, M. Lewenstein, and M. A. Martin-Delgado, Phys. Rev. Lett. 103, 035301 (2009).

[14] R. A. Williams, S. Al-Assam, and C. J. Foot, Phys. Rev. Lett. 104, 050404 (2010).

[15] J. Struck, C. Ölschläger, M. Weinberg, P. Hauke, J. Simonet, A. Eckardt, M. Lewenstein, K. Sengstock, and P. Windpassinger, Phys. Rev. Lett. 108, 225304 (2012).

[16] K. Jimenez-Garcia, L. J. LeBlanc, R. A. Williams, M. C. Beeler, A. R. Perry, and I. B. Spielman, Phys. Rev. Lett. 108, 225303 (2012).

[17] P. Hauke, O. Tieleman, A. Celi, C. Ölschläger, J. Simonet, J. Struck, M. Weinberg, P. Windpassinger, K. Sengstock, M. Lewenstein, and A. Eckardt, Phys. Rev. Lett. 109, 145301 (2012).
[18] G. Mazzucchi, L. Lepori, and A. Trombettoni, J. Phys. B 46, 134014 (2013).

[19] M. Aidelsburger, M. Atala, M. Lohse, J. T. Barreiro, B. Paredes, and I. Bloch, Phys. Rev. Lett. 111, 185301 (2013).

[20] J. Struck, M. Weinberg, C. Ölschläger, P. Windpassinger, J. Simonet, K. Sengstock, R. Höppner, P. Hauke, A. Eckardt, M. Lewenstein, and L. Mathey, Nat. Phys. 9, 738 (2013).

[21] T. P. Polak and T. A. Zaleski, Phys. Rev. A 87, 033614 (2013).

[22] N. Marzari and D. Vanderbilt, Phys. Rev. B 56, 12847 (1997); N. Marzari, A. A. Mostofi, J. R. Yates, I. Souza, and D. Vanderbilt, Rev. Mod. Phys. 84, 1419 (2012).

[23] J. Ibañez-Azpiroz, A. Eiguren, A. Bergara, G. Pettini, and M. Modugno, Phys. Rev. A 87, 011602 (2013); 88, 033631 (2013).

[24] K. L. Lee, B. Grémaud, R. Han, B.-G. Englert, and C. Miniatura, Phys. Rev. A 80, 043411 (2009).

[25] The potential can be exactly mapped into that used in our previous work [23], by a counterclockwise rotation by $90^{\circ}$ of the axes $\left(\boldsymbol{e}_{x} \rightarrow \boldsymbol{e}_{y}, \boldsymbol{e}_{y} \rightarrow-\boldsymbol{e}_{x}\right)$. The same potential is also equivalent to that used by Stanescu et al. [4] (by posing $V_{0}=4 s$ ) and by Shao et al. [2] (except for an irrelevant shift of the coordinates).

[26] J. Ibañez-Azpiroz, A. Eiguren, A. Bergara, G. Pettini, and M. Modugno (unpublished).

[27] We have verified that values of $t_{0},\left|t_{1}\right|$, and $\varphi$ obtained with the two methods allow us to reproduce the exact spectrum with great accuracy in the proper tight-binding regime, $s \gtrsim 3$; a detailed discussion will be presented elsewhere [26].

[28] G. Jotzu, M. Messer, R. Desbuquois, M. Lebrat, T. Uehlinger, D. Greif, and T. Esslinger, arXiv:1406.7874. 